\title{
Asking the next generation: The implementation of pre-university students' ideas about physics laboratory preparation exercises.
}

\author{
K. Dunnett \\ Nordita, KTH Royal Institute of Technology and Stockholm University, \\ Roslagstullsbacken 23, SE-106 91 Stockholm, Sweden $\ddagger$

\section{P. A. Bartlett} \\ Department of Physics and Astronomy, University College London, Gower Street, \\ London WC1E 6BT, UK \\ E-mail: paul.bartlett@ucl.ac.uk
}

\begin{abstract}
It was planned to introduce online pre-laboratory session activities to a first-year undergraduate physics laboratory course to encourage a minimum level of student preparation for experiments outside the laboratory environment. A group of 16 and 17 year old laboratory work-experience students were tasked to define and design a pre-laboratory activity based on experiments that they had been undertaking. This informed the structure, content and aims of the activities introduced to a first year physics undergraduate laboratory course, with the particular focus on practising the data handling. An implementation study showed how students could try to optimise high grades, rather than gain efficiency-enhancing experience if careful controls were not put in place by assessors. However, the work demonstrated that pre-university and first-year physics students can take an active role in developing scaffolding activities that can help to improve the performance of those that follow their footsteps.
\end{abstract}

Submitted to: Phys. Educ.

$\ddagger$ K. Dunnett undertook this research study when working at UCL prior to moving to Nordita 


\section{Introduction}

University College London (UCL) undertakes the training of physicists in experimental techniques. All first year physics undergraduate students are exposed to experimental physics. Astrophysicists, Theoreticians, Medical Physicists and Applied Physicists are all trained together for the first term. The first-year physics teaching laboratory's activities focus on training all students so that they can appreciate the difference between research-level research and the 'guided demonstrations' that they are exposed to during their pre-university studies. The aim is to start to develop the knowledge, skills and attributes of an experimental physicist. Students are encouraged to think for themselves and are made to challenge their assumptions at every point during an experiment. In addition, they are given the opportunity to modify their experiments so that they can investigate their own ideas based on their own observations. Experimental scripts are not considered to be a structure to slavishly follow but are to be used as the seed for further investigation. This deviates from a more traditional teaching-laboratory course structure that uses experimentation just to supply a physical realisation of a concept presented in a lecture series. The experiments may be considered to lie somewhere between inquiry and discovery type [1] or between guided and structured inquiry [2, 3] depending on the classification system used. The emphasis in UCL's physics laboratories is to give students new experimental skills that cannot be gained via theoretical treatments alone. This brings our methods in-line with those that that attempt to move away from the more traditional guided demonstration model of physics teaching laboratories $[4,5]$.

A full-cohort survey of first year UCL undergraduate physics students conducted at the end of their second term of practical laboratories in academic year 2014-15 revealed that only half of the students claimed to read the scripts provided before the start of a four-session experiment, so had to spend some of the restricted laboratory time reading the laboratory script (lab script). It was proposed to introduce Moodle based pre-laboratory (pre-lab) activities that would motivate students to read the lab scripts before starting the practical sessions by requiring them to complete a short quiz that aimed to draw their attention to particular aspects of the experiment. Moodle is an open-source learning management system that can be used to set, manage and mark quizzes that teaching staff can devise prior to a course taking place[6]. It can also be used to monitor how students use Moodle.

Instead of the laboratory staff, academics and post-graduate demonstrators creating pre-lab activities without considering students' needs, use was made of a group of c. 20 laboratory work experience students at UCL who were about one academic year behind the target first year undergraduate students. This meant that the needs of students prior to their joining UCL could be identified and, perhaps, met in the future by using the experience of these students to help design pre-lab activities.

The ideas gained from the work experience students were used to develop Moodle based pre-lab activities for five experiments (each comprising $4 \times 3.5$ hour experimental sessions which should give students ample time for repeats and exploration of the physics 
underlying the experiment) conducted in the first two terms by first year undergraduate physics students. The undergraduate students were asked for their reactions to the pre-lab activities at the end of the second term.

The work experience students' suggestions were focussed on the details that would increase the efficiency of performing or undertaking the experiments and subsequent analysis, a feature that was different to UCL demonstrators' initial ideas about developing understanding of the physics that students would encounter in the experiment before starting, but in keeping with research on the purpose of pre-lab activities $[7,8,9]$.

The remainder of the paper details, in order, the task of the work experience students and the insights gained, the initial implementation and observations made about completion strategies. Some results of an end of module student opinion questionnaire are also included and discussed. The value of the experience and suggestions for other implementations are discussed in the conclusions.

\section{Pre-university students defining and designing pre-lab activities}

A group of seventeen 16-17 year old students conducted four experiments over the first four days of their work experience week without any pre-lab activities, giving them an insight into experiments in an university context. On the fifth day, their task was to define what they thought a generic pre-lab activity should do to help students conduct university level physics experiments and what types of questions it should contain. They were also asked to consider marking methods and designed a pre-lab activity for one of the experiments that they had completed during the week.

\subsection{Task and background information supplied}

The briefing given to the work experience students' task was as follows:

"A prep-task [pre-lab] is an activity completed independently before the start of a more complicated, usually practical, activity. Today's task is to design a prep-task for one of the experiments done this week. Existing prep-tasks for first year undergraduate labs at various institutions involve a range of activities such as: reading the lab script before the lab session; reading the script and then writing a brief method and completing a simple calculation; activities to develop understanding of the equipment used in the lab; activities to teach technical skills such as understanding a particular type of plot; introduction to physics concepts."

The students worked in four groups (three groups of four and one of five) and were asked to consider some of the practical aspects as well as the purpose of a pre-lab activity, but the demonstrators involved in running the day tried not to guide the students towards any particular format of pre-lab activity beyond bringing their attention to some of the types of questions that could be created as part of an online quiz. It was also pointed out that both students and assessors would have other demands on their 
time prior to laboratory sessions. They were also asked to think carefully about what they hoped students would get out of each element of the prep-task. After each group had designed their pre-lab activities, they presented their general ideas about pre-lab activities and these were discussed by the whole group.

\subsection{Purpose of a pre-lab activity}

The four groups identified several key aims of any pre-lab activity with the target of preparing students for experiments, even to the level of students being able 'to walk into a lab and perform that experiment without any help'. While this might not be possible in practice and would always depend on the motivation of the individual students, a successful pre-lab activity should improve students' efficiency when in the lab. It must be noted that students may need inducements to ensure that they compete pre-laboratory activities as they may not consider improvements in efficiency as sufficient motivation to undertake the tasks [10].

Several themes arose in all of the groups: a need for general preparation and consequent improved efficiency during laboratory sessions; familiarisation with the theory and equations necessary for data analysis and an introduction to new or experiment-specific equipment. Two other, rather different, aspects also appeared: an introduction to the safety considerations surrounding the particular experiment and enabling motivation for further investigation of the experiment - equipment or theory before entering the lab. The first of these depends very much on the exact level of risk associated with the experiment in question. The second depends almost entirely upon the motivation and state of epistemological development of individual students. Indeed, it has been suggested that students ideas about themselves as science learners are more likely to influence their efforts to learn science than their ideas about a distant 'formal science' community that they do not consider themselves to be part of [11].

\subsection{Content of pre-lab activity}

The content of a pre-lab activity was identified by the students both generally and for specific experiments. Three groups created pre-lab activities for an experiment that they had conducted earlier in the work experience week; they were therefore considering the type of pre-lab activity that they would have liked to have completed prior to the experiment. The fourth group (consisting of five students) chose to write a pre-lab activity for an experiment that they were not familiar with, but is one of the experiments that all first year undergraduate physics students undertake in the first term at UCL.

Several features appeared in different groups' descriptions of the aim of a pre-lab activity. In the pre-lab activities that were finally implemented, common features of the definitions and the example pre-lab activities that the work experience students created were used together.

It was recognised that familiarity with an experiment's procedure might be difficult

to obtain without either a detailed 'simulation' or doing the actual experiment and 
learning from direct experience, although it would be possible to have students analyse data obtained from previous experimental groups. However, some familiarity with the experiment could be obtained either via students labelling a diagram or photograph or by them matching the pieces of equipment with their use in an experiment. This could also include activities such as correctly interpreting a standard labelling mechanism, e.g. the coloured bands on resistors.

The particular aspects that were highlighted by the work experience students and included in the subsequent implementation were aimed at gaining familiarity with the equations that they would be using for analysing the data obtained and the uncertainty propagation that formed a significant part of the data analysis. The first was a matter of identifying which equation(s) in the script, usually present as part of a derivation, should be recorded in the laboratory notebook. In previous years, there had been a tendency for undergraduate students to copy large portions of the 'theory' section of the scripts directly into their laboratory notebooks without considering which equations or diagrams, particularly those detailing technical aspects of equipment, and any associated text, were really relevant to the experiment. This had been noted to contribute to student inefficiency in their first experimental session. The second consideration aimed to give the students some expectation of the form of the equation they would need for their uncertainty propagation calculations.

\subsection{Potential implementation methods}

In general, a compulsory quiz of some sort was suggested as the submission or test mechanism of the pre-lab activity for which immediate automated feedback could be provided. Some of the most interesting aspects considered in the whole group discussion related to the time for completion and marking, and whether completing the quiz would contribute to the mark for the lab course. This last consideration lead to some serious and lively discussion between the groups, eventually coming to the conclusion that a mark should be given for the pre-lab activity, but this mark might not necessarily contribute to the overall grade for the laboratory course.

All groups agreed that in order to complete any pre-lab activity successfully the students would have to read the experiment's lab script. However, this would probably be the most time consuming part of the entire activity, unless some use of online simulations was recommended, but the use of external simulations could, in their opinion, never be made compulsory. In terms of something that would be marked, a compulsory multiple-choice quiz, easily organised using online learning environments, was the usual suggestion. The main advantages of a multiple-choice quiz are that the marking and feedback can be automated, thus requiring no direct time commitment from demonstrators before or during laboratory sessions.

They also considered that reading the script and completing the quiz should take 30-60 minutes, making it long enough to be a worthwhile exercise, but not so time consuming as to become a serious addition to students' workloads. This is in keeping 
with a preferred 30 minute timescale for a rather different type of pre-lab activity in an engineering context [12], and seems reasonable given the length of the laboratory scripts (4-15 pages of content) with the longer scripts including many more diagrams.

The work experience students recognised that familiarity with the information in the lab scripts could be easily tested, but further exploration could only be encouraged. In principle, the quizzes could also contain additional information about the experiment and the students tested on their understanding of these concepts. Given that UCL physics students are encouraged to explore beyond the limits of the scripts, to direct students towards specific additional resources, or introduce a particular nuance of the experiment before students enter the laboratory could have the unwanted effect of basically providing an extended script and so individual investigations going beyond them might be limited to pre-determined areas. The pre-lab activities were therefore restricted to the content of the scripts.

\section{Pre-lab activity implementation}

Based on the understanding gained from the ideas of students slightly younger than those for whom the pre-lab activities were designed, some general elements are discussed along with students' reactions.

\subsection{Moodle quiz content}

For all experiments, the pre-lab Moodle quiz activities were written so that they were significantly easier if the script had been read beforehand and most of the information required would be either in the lab script or included in the question. An exception was uncertainty propagation tasks where the aim was to check that students were aware of the form of the equation that they would need to use. The multiple choice format restricted the type of questions that could be asked and the depth of understanding that could be assessed. Uncertainty propagation was taught in a separate lecture course running parallel to the lab courses, but no formulae were included in the lab script.

There were two general types of pre-lab activities depending on the type of experiment. Most of the experiments in the first year lab course focus on the process of conducting an experiment and exploring the extent to which the experimental data agreed with the theory presented in the lab script. In such cases, the data analysis formed the most important part of the experimental process and the quiz generally required students to correctly identify the equations that would be used for manipulating the data. First, they would have to identify and use the correct equation in the script to complete a calculation on some sample data effectively giving them an opportunity to practice the basic data analysis; then, they would have to recognise the most important equation for propagating the uncertainties in the measured values through to the uncertainty on the final quantity (which was not in the script). If calculations were performed on data obtained from, say, a plot, an example figure was often included in 
the question, even though students were not asked to extract any information from it. Students were therefore exposed to a (sometimes idealised) version of what they could be expected to obtain at some point during the experiment.

An example of a Moodle quiz question is shown in Figure 1, demonstrating how data from previous students' work could be used to train future students. It shows an ellipse that is produced by a laser beam connected to a rotating mechanical system that is displaying a damped response to a sinusoidal actuating force. The ellipse is produced by mapping the driving oscillation and the resulting motion to the planar movement of the laser spot in the $x$ and $y$ directions respectively. The students would need to find and use the correct equation from the lab script to solve this problem.

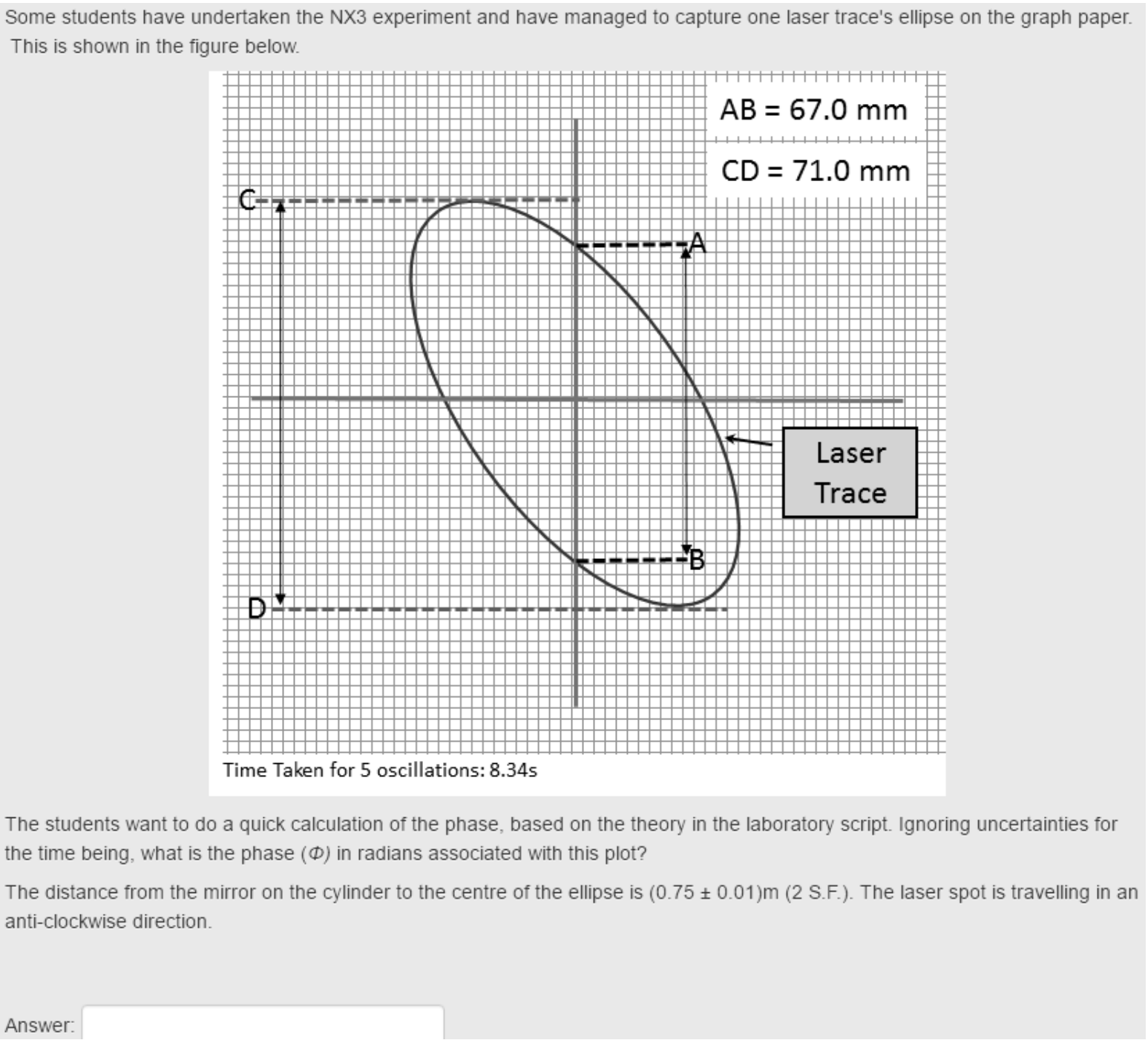

Figure 1. Example Moodle pre-lab question which tests a student's understanding of how a laser-based measurement system's graphical output can be related to the mechanical oscillation of a torsional pendulum that it is measuring. Students can give an answer to this question through the use of the lab script 


\subsection{Students completion strategies}

Although the very nature of a pre-lab activity is for it to be attempted before entering the labs, in the first year of introduction, there was no hard deadline implemented and the quiz could be attempted up to three times. It was then possible to see how the students behaved when confronted with such a pre-knowledge test, as we could monitor how the questions were tackled via the Moodle Reports system. The students were unaware that such an analysis would take place. It was hoped that it would be possible to analyse student behaviours when confronted with a pre-knowledge test presented in this fashion.

There were two main effects of this freedom of when to complete and the opportunity for multiple attempts. The undergraduate students often took the prelab activity more than once to obtain $100 \%$ although the contribution to the module grade was very small. Some students took only a few seconds to complete the quiz, getting zero and then made a second attempt a minute or two later getting full marks as a result of remembering the correct answers from their previous attempt. It was clear that students using such a strategy were more motivated by getting full marks than understanding the experiment.

Undertaking the pre-lab activity within the first session of an experiment was common, thereby using lab time to learn things that could help them to get a good grade. This was not what the pre-lab test was intended for and the contribution to the module grade was kept intentionally low $(5 \%)$ to discourage this sort of behaviour. Another student behaviour that was observed was returning to the pre-lab activity to extract the equation for propagating the uncertainties in a later session. In this case, the students were using the quiz as a resource rather than a self-checking system to aid understanding.

This is consistent with previous studies that suggest that students are strategic learners who will allocate their time and focus on what they will believe will give them the best grades with the least effort [13]. It has been found that students will attempt to adapt to any new assessment methodology to achieve this even if it means trading understanding for grades.

All three problems can be circumvented through setting up the online quiz so that the pre-lab activity becomes unavailable shortly before the start of the first experimental session and by preventing multiple attempts. In academic year 2016-2017, the students were allocated to specific groups that restricted their access to the Moodle quiz so that the attempt window was reduced to one week before the start of a new experiment. This prevented them from completing the task after they had started the practical sessions. This resulted in a number of students asking if they could undertake the pre-knowledge test later in the term, as they had not completed it in the allotted time. This was not allowed in any circumstances as the rules regarding the quiz completion had been made clear in briefings at the start of the term and in the course documentation. 


\subsection{Students' reactions}

At the end of the second term of the 2016-2017 session, the student cohort were asked what they thought of the pre-lab activities (see Figure 2 and Figure 3). Most of the 40 students that responded to the questionnaire seemed to think that the pre-lab activities were useful preparation for the up-coming experiments but even more considered that they were relevant to the experiments.

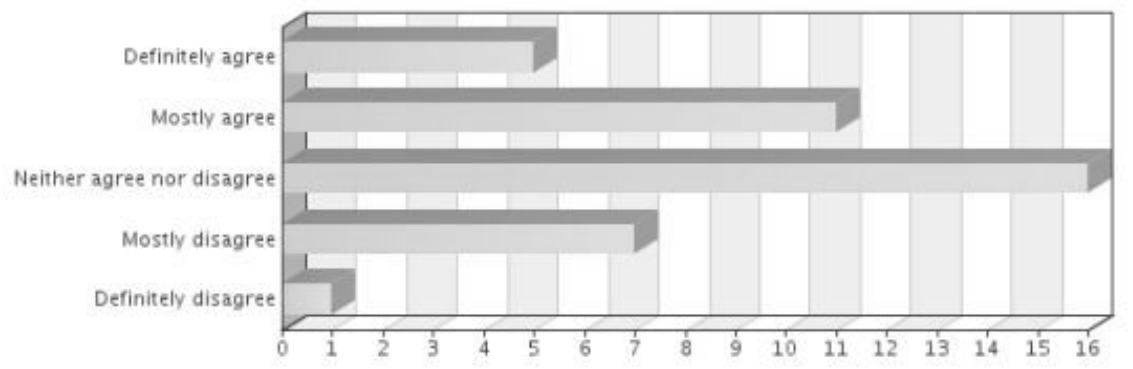

\section{Frequency table}

\begin{tabular}{|c|c|c|c|}
\hline Items & $\begin{array}{l}\text { Absolute } \\
\text { frequency }\end{array}$ & $\begin{array}{l}\text { Relative } \\
\text { frequency }\end{array}$ & $\begin{array}{l}\text { Adjusted } \\
\text { relative } \\
\text { frequency }\end{array}$ \\
\hline Definitely agree & 5 & $11.63 \%$ & $12.5 \%$ \\
\hline Mostly agree & 11 & $25.58 \%$ & $27.5 \%$ \\
\hline Neither agree nor disagree & 16 & $37.21 \%$ & $40 \%$ \\
\hline Mostly disagree & 7 & $16.28 \%$ & $17.5 \%$ \\
\hline Definitely disagree & 1 & $2.33 \%$ & $2.5 \%$ \\
\hline Sum: & 40 & $93.02 \%$ & $100 \%$ \\
\hline Not answered: & 3 & $6.98 \%$ & - \\
\hline
\end{tabular}

Figure 2. Questionnaire question asked of students in 2017: The pre-lab activities were good preparation for the experiment? 


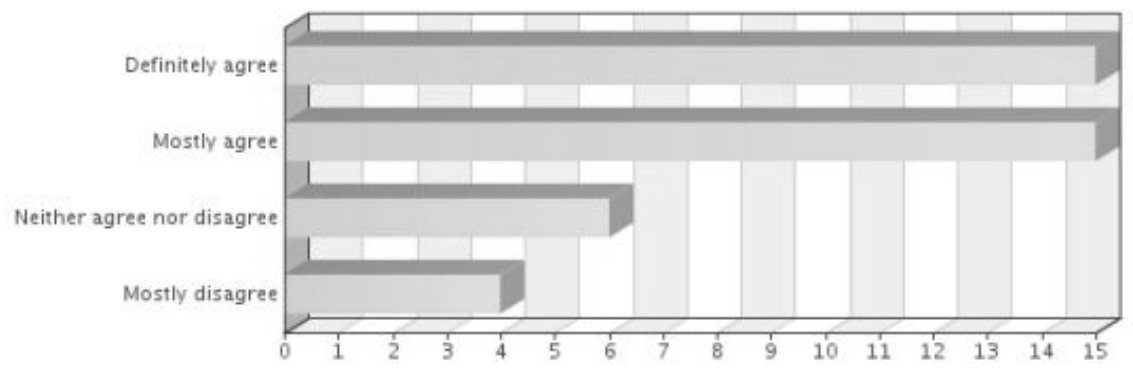

Frequency table

\begin{tabular}{llll}
\hline Items & $\begin{array}{l}\text { Absolute } \\
\text { frequency }\end{array}$ & $\begin{array}{l}\text { Relative } \\
\text { frequency }\end{array}$ & $\begin{array}{l}\text { Adjusted } \\
\text { relative } \\
\text { frequency }\end{array}$ \\
\hline Definitely agree & 15 & $34.88 \%$ & $37.5 \%$ \\
Mostly agree & 15 & $34.88 \%$ & $37.5 \%$ \\
Neither agree nor disagree & 6 & $13.95 \%$ & $15 \%$ \\
Mostly disagree & 4 & $9.3 \%$ & $10 \%$ \\
\hline Sum: & 40 & $93.02 \%$ & $100 \%$ \\
\hline Not answered: & 3 & $6.98 \%$ & - \\
\hline Total answered: 40 & & &
\end{tabular}

Figure 3. Questionnaire question asked of students in 2017: The pre-lab activities were relevant to the experiment?

Given that the pre-lab activities that these students undertook were the original implementation, this was important confirmation that by involving the younger work experience students in the design of the pre-lab activities, the activities could be tailored to the target audience without multiple iterations being required for fine tuning. Taken as an indication of the students' satisfaction with the pre-lab activities as a component of the laboratory course, the recently introduced pre-lab activity in its original form is meeting the students' needs, and understood by the students as well as other assessment components that have been developed over the course of several years.

\section{Conclusions}

On their own, the work experience students' ideas provided an interesting insight into the students' expectations and were also very useful for constructing the pre-lab activities as they helped focus the type of questions asked and the aspects of the experiments that were covered in particular detail. Since these students were from London schools, their educational experience (England's GCSE and A-Level system) was representative of about two thirds of the UCL undergraduate cohort, making their ideas relevant to the student body for whom the quizzes were intended.

By observing students' completion behaviour, the utility of an online preparatory quiz without a rigidly enforced completion deadline is seen to be questionable. Future iterations of pre-lab questionnaires at UCL have enforced a strict deadline; to do so effectively requires efficient communication of the deadlines to the students. 
By engaging with students with similar experiences to those who were expected to complete the pre-lab questionnaires as part of their laboratory courses, preparatory work beyond simply reading the scripts could be encouraged that would stand the students in good stead and inform them accurately of some aspects of the experiments before they entered the laboratory. Further, identifying the students' needs and tailoring the activities appropriately resulted in reasonable student satisfaction without a lengthy iteration process. It must be stressed that the four session format of the experiments allowed plenty of time for students to develop their understanding of the relevant physics concepts through exploring the experimental system. The pre-lab activities were aimed at improving the students' efficiency in identifying what information in the lab scripts was actually relevant to the immediate experimental needs rather than just being background detail.

Much can be learned by utilising pre-university students when designing new components of courses, especially for first year. This should have great potential when introducing new activities as younger students with a similar background will provide a more realistic gauge of the target students' expectations, knowledge and motivations compared with students who have already completed the course and demonstrators. Meanwhile, observing the completion data of Moodle quizzes can help understand students' behaviours and can be used to gauge student motivations and may enable the introduction of suitable controls to prevent (to an extent) unwanted behaviours.

\section{Acknowledgments}

We would like to thank the UK A-Level students that attended the 2015 UCL Work Experience programme and acted as a 'focus group' for this work.

[1] Daniel S Domin. A review of laboratory instruction styles. Journal of chemical education, 76(4):543, 1999.

[2] Randy L Bell, Lara Smetana, and Ian Binns. Simplifying inquiry instruction. The Science Teacher, $72(7): 30-33,2005$.

[3] Laura B Buck, S Lowery Bretz, and Marcy H Towns. Characterizing the level of inquiry in the undergraduate laboratory. Journal of College Science Teaching, 38(1):52-58, 2008.

[4] Susan Howitt, Anna Wilson, Kate Wilson, and Pam Roberts. Please remember we are not all brilliant: undergraduates experiences of an elite, research-intensive degree at a research-intensive university. Higher Education Research 83 Development, 29(4):405-420, 2010.

[5] N. G. Holmes, Jack Olsen, James L. Thomas, and Carl E. Wieman. Value added or misattributed? A multi-institution study on the educational benefit of labs for reinforcing physics content. Phys. Rev. Phys. Educ. Res., 13:010129, May 2017.

[6] Moodle Features. https://docs.moodle.org/33/en/Features/, 2017. [Online; accessed 12Sept-2017].

[7] Orla C Kelly and Odilla E Finlayson. Providing solutions through problem-based learning for the undergraduate 1st year chemistry laboratory. Chemistry Education Research and Practice, 8(3):347-361, 2007.

[8] Juan-Antonio Llorens-Molina. Design and assessment of an online prelab model in general chemistry: A case study. Journal of the Research Center for Educational Technology, 4(2):15-31, 2009 . 
[9] Norman Reid and Iqbal Shah. The role of laboratory work in university chemistry. Chemistry Education Research and Practice, 8(2):172-185, 2007.

[10] Marissa Rollnick, Stella Zwane, Mina Staskun, Sandra Lotz, and Gail Green. Improving prelaboratory preparation of first year university chemistry students. International Journal of Science Education, 23(10):1053-1071, 2001.

[11] William A Sandoval. Understanding students' practical epistemologies and their influence on learning through inquiry. Science Education, 89(4):634-656, 2005.

[12] Chuin-Shan Chen, Shang-Hsien Hsieh, Sheng-Chin Chuang, and Shiu-Shin Lin. A prelab tutoring system for strength of materials experiment. Computer Applications in Engineering Education, 12(2):98-105, 2004.

[13] Graham Gibbs. 2 How assessment frames student learning. Innovative assessment in higher education, page 23, 2006. 